\title{
La « haalpularisation » ou la mise en discours de la culture et de la langue pulaar au Sénégal
}

Processus et enjeux

The "Haalpulaarization" or the "Enwordment" of Pulaar Language and Culture in Senegal. Processes and Stakes

\section{El Hadji Abdou Aziz Faty}

\section{OpenEdition}

\section{Journals}

Édition électronique

URL : http://journals.openedition.org/etudesafricaines/18011

DOI : 10.4000/etudesafricaines.18011

ISSN : 1777-5353

\section{Éditeur}

Éditions de l'EHESS

\section{Édition imprimée}

Date de publication : 1 avril 2015

Pagination : 67-84

ISSN : 0008-0055

\section{Référence électronique}

El Hadji Abdou Aziz Faty, «La « haalpularisation » ou la mise en discours de la culture et de la langue pulaar au Sénégal », Cahiers d'études africaines [En ligne], 217 | 2015, mis en ligne le 31 mars 2017 consulté le 01 mai 2019. URL : http://journals.openedition.org/etudesafricaines/18011 ; DOI 10.4000/etudesafricaines. 18011 


\title{
La « haalpularisation » ou la mise en discours de la culture et de la langue pulaar au Sénégal
}

\author{
Processus et enjeux
}

Les revendications identitaires et culturelles des Haalpulaar au Sénégal reposent en général sur deux enjeux majeurs : la défense du territoire (le Fouta sénégalais) et celle de la langue (le pulaar). Le territoire et la langue sont l'un et l'autre considérés comme des critères de différenciation et de démarcation. Ils font également l'objet de représentations débouchant sur des tentatives d'homogénéisation de la communauté pulaarophone. Cet article rendra compte des différentes formes d'homogénéisation mises en œuvre avant d'expliquer les contextes sociopolitiques dans lesquels elles trouvent leur ancrage. Les discours épilinguistiques (Canut 2007b) sur le pulaar, loin d'être aboutis, sont en perpétuelle construction dans l'interaction en fonction de l'interlocuteur, de la situation de communication et des enjeux de pouvoir, etc.

Dans un de ces articles, Fiona Mc Laughlin (1995) avançait la thèse selon laquelle la «haalpularisation », c'est-à-dire les mouvements de revendication identitaire des Haalpulaar au Sénégal, n'est ni plus ni moins qu'une réponse à la "wolofisation ». L' «identité » haalpulaar telle qu'elle a été perçue ou telle qu'elle est défendue par «ses partisans », ne saurait être comprise en l'isolant du processus de «wolofisation » qui l'a rendue (et la rend toujours) possible. En effet, à l'instar de leurs frères de l'autre rive du fleuve, c'est-à-dire les Haalpulaar de la Mauritanie, ceux du Sénégal se sont aussi considérés exclus du système politique post-indépendance du fait de la supposée mainmise des Wolof sur le pouvoir. Cette "hégémonie» des Wolof est d'autant plus prégnante que toutes les instances de décision politiques, économiques, commerciales ou financières se concentrent dans des zones traditionnellement wolofones (Dakar, Thiès, Saint-Louis). C'est ce qui a été, sans nul doute, à l'origine de la conscience identitaire haalpulaar qui se traduit par la mise en place de mouvements de défense de l'identité, de la culture, à travers la langue pulaar. Ces mouvements sont générateurs de discours portant sur la crainte de perdre sa langue ainsi que sa culture. 
Mais la «wolofisation» reste-t-elle la seule explication de ce phénomène de « haalpulaarization» pour emprunter le terme de F. Mc Laughlin (1995).

L'effacement des frontières" (Augé 2009), dû à la "mobilité surmoderne $»^{2}$, et la libre circulation des personnes ont provoqué de profonds changements sociaux qui suscitent des sentiments d'inquiétude chez les locuteurs pulaarophones. Leurs discours sur la défense de la langue et de la culture pulaar constituent dès lors un prétexte pour arriver à aborder certaines questions cruciales, telles que le partage des terres, les redistributions économiques, etc. Les changements sociaux, politiques et économiques participent donc à l'élaboration de la réalité à travers la création de récits fantasmés sur le territoire et sur la langue. Dans le cadre de cet article, nous nous focaliserons sur l'analyse de ces récits tout en soulignant leur caractère performatif dont la force illocutoire vise à « mobiliser» les membres de la communauté dans la cause défendue. Ces discours seront donc considérés comme des actes, des «pratiques sociales» (Boutet 2008) ainsi que des « réponses à des problèmes empiriques des agents ${ }^{3}$.

\section{La wolofisation ${ }^{4}$}

Nous avons emprunté ce concept de wolofisation à un certain nombre de chercheurs comme M. Diouf (1994), O’Brein (1998), Mc Laughlin (2001). Du point de vue politique, la wolofisation ne serait qu'un processus d'intégration «par le bas » (Smith 2010) face au modèle d'intégration politique dite «par le haut» de l'État colonial et postcolonial sénégalais. Face donc à l'échec de la politique de francisation, la wolofisation s'impose de plus en plus comme une nouvelle forme d'identification nationale. Et ce, grâce aux «dynamiques sociales » qui, selon É. Smith (ibid.), ont joué (et continuent

1. J'utilise ici l'expression « effacement des frontières » mais, tout comme M. AUGÉ (2009), je considère que les frontières ne s'effacent jamais, mais se redessinent.

2. «La mobilté surmoderne s'exprime dans les mouvements de population (migrations, tourisme, mobilité professionnelle), dans la communication générale instantanée et dans la circulation des produits, des images et des informations. Elle correspond au paradoxe d'un monde où l'on peut théoriquement tout faire sans bouger et où l'on bouge pourtant » (AUGÉ 2009: 7-8).

3. Voir les théoriciens russes Vigotsky, Vorochilov et Bakhtine.

4. «Wolofisation : action de wolofiser, de se wolofiser. Là se manifeste le premier indice d'un processus de "wolofisation" de la production littéraire de Sembène (Tine 1985). La wolofisation du français dans les exemples suivants s'explique par le fait que l'intention sémantique est conçue en wolof avant d'être rendue en français (DAFF 1993a). Évidemment tout observateur attentif et impartial ne peut manquer d'être frappé par la forte présence wolof dans la Société d'Accaparement [...], à tel point que l'on a pu parler de "processus de wolofisation" de la société toute entière (NDIAYE 1996). En effet, dans leur terroir du Baol, les Sérère sont entraînés dans une "wolofisation" qu'accélère encore l'exode rural [...] (Walfadjri 12/04/1996) », Corréard Géneviève N’DIAYE \& équipe du projet IFA, 2006, Les mots du patrimoine : le Sénégal, Archives contemporaines, p. 573. 
d'ailleurs de jouer) un rôle important dans le processus de la formation d'une identité sénégalaise. À en croire D. C. O'Brein (1998 : 27), l'identification au wolof n'est rien d'autre qu'un processus, lequel est en rapport avec des paramètres tels que la migration, l'urbanisation, la religion, etc. :

«Wolof identification is perhaps best seen as a process, one which relates to a range of subjects : urbanization, migration, religion, statehood. There are no fixed ethnic boundaries [...]. »

C'est ainsi qu'au Sénégal, la langue wolof reste sans nul doute la langue la plus parlée, non pas du fait d'une supériorité numérique « ethnique » mais de sa croissance exponentielle. La particularité de cette langue est que son expansion dépasse de loin les frontières ethniques: il n'y a pas que les Wolof qui utilisent cette langue. C'est une langue parlée jusque dans des zones où elle n'avait traditionnellement pas cours. Beaucoup de chercheurs ont montré comment cette langue s'est répandue à travers le pays, en «menaçant » les «bastions » des autres langues nationales. Dans leur étude consacrée à la vie des langues au Sénégal, M. Dreyfus et C. Julliard (2001 : 667) ont montré comment le wolof domine jusque dans des zones comme la Casamance par exemple, où les langues du Sud telles que le diola ou le mandingue ont toujours régné en maître.

«L'expansion du wolof y est récente, son usage progresse du centre ville, commercial et administratif, à la périphérie, au mode de vie proche de celui du monde rural avoisinant ; cette langue tend à prendre la position dominante dans la nouvelle configuration multilingue.»

Cette langue connaît en effet une expansion telle que, du point de vue sociolinguistique, le concept de wolofisation s'est imposé sans difficulté. Bon nombre de linguistes comme Fiona Mc Laughlin (2001) n'hésitent pas à recourir à ce néologisme pour en marquer la prégnance. On peut le définir comme étant le processus de propagation ou d'expansion de la langue wolof dans le paysage sociolinguistique sénégalais. C'est le passage d'un état de vernacularisation de cette langue à celui de véhicularisation. Autrement dit, le wolof n'est plus la langue d'une « ethnie » donnée, mais une langue qui est utilisée presque par tout un pays, une lingua franca:

"The language that has filled the role of lingua franca in Senegal is Wolof» (Mc Laughlin 2001 : 159).

«Wolof has continued to spread as a spoken language, a lingua franca of commercial contact and of urbanization [...]»(O'Brein $1998: 29)$.

La langue pulaar face à la langue wolof et à d'autres «langues hégémoniques»

Beaucoup de non-Wolof (des intellectuels principalement) voient dans la «wolofisation », l'expansion d'une langue susceptible de «tuer» les leurs. 
On retrouve ces idées même dans les travaux de certains linguistes sénégalais comme Papa Alioune Ndao qui qualifie de " glottophagique » la relation inégale entre le wolof et les autres langues du pays. Ce terme de «glottophagie » est d'ailleurs emprunté à l'école sociolinguistique de Rouen. Selon Ndao (2009), la «glottophagie c'est lorsqu'une langue arrive à en avaler une autre de par son dynamisme ». Le verbe avaler utilisé par le sociolinguiste témoigne non seulement de sa conception naturaliste et organiciste de la langue, mais aussi d'une vision diglossique dont les limites ont été attestées par un certain nombre de chercheurs parmi lesquels Cécile Vigouroux (2009). Dans un de ses articles, cette dernière, contrairement à d'autres chercheurs, s'appuie sur la notion de fonctions communicatives des langues. Pour elle, il n'y a pas lieu de parler de langue en danger ou de diglossie quand les deux langues n'ont pas les mêmes fonctions communicatives. À l'issue d'une enquête de terrain menée à Cap Town auprès de populations d'immigrés venus de différentes zones de l'Afrique francophone, elle arrive à la conclusion que l'anglais (langue dominante à Cap Town), à aucun moment des interactions, n'est considéré comme une langue menaçante par ces populations migrantes qui l'utilisent même entre eux comme langue véhiculaire. Elle a donné l'exemple des anciens migrants qui s'adressent en anglais aux nouveaux venus dans le but de marquer leur statut d'anciens et de montrer leur intégration dans le pays hôte. Dans cette situation, l'utilisation de l'anglais est, selon la chercheure, indexicale dans la mesure où elle rend compte de la stratification sociale des migrants. D'où la prise en compte de la complexité des dynamiques socioculturelles :

«Linguistic studies of globalization such as Crystal, Fishman, or Maurais [...] "have often slip[ed] uneasily between description and prediction" [...], and run the risk of misrepresenting complex local linguistic dynamics » (Vigouroux 2009: 249).

Il ne serait sans doute pas abusif de transposer l'analyse de C. Vigouroux dans le contexte sénégalais et se demander si, réellement, le locuteur lambda pulaarophone à Dakar ou à Saint-Louis se sent menacé par la langue wolof (dont il connaît implicitement ou explicitement la ou les fonctions communicatives) au point de refuser d'en faire usage. D'après mes enquêtes et observations sur le terrain (Faty 2011), j'ai montré le rôle des paramètres autres qu'ethniques (l'affect, le désir, les rapports de pouvoir, etc.) dans les positionnements et les pratiques langagières des sujets parlants. Ce sont ces positionnements de pouvoir dans le langage que les sociolinguistes de la diglossie et des langues en danger semblent avoir oubliés au point d'user de verbes violents comme «tuer» ou « avaler» pour décrire certains phénomènes linguistiques. Ces mêmes termes apparaissent dans les discours des militants. C'est ainsi que dans le discours d'un militant pulaarophone avec qui je me suis entretenu est apparu un néologisme de type mod pinalaagu qui pourrait être traduit en français par «le processus d'avalement des cultures ». Ce néologisme a été créé à partir du verbe modde : «avaler» 
en français et de pinal, «culture », suivi du suffixe aagu pour introduire la notion de processus. Cette expression néologique témoigne d'un certain état d'esprit dans lequel sont plongés les tenants des discours essentialistes sur les langues, état d'esprit dénotant une certaine vision conflictuelle et violente de la vie des langues.

«RPC ${ }^{5}$ : Tidiane Ann lui-même l'a dit ah / il a dit heu en s'adressant aux Fulbe "notre langue a été avalée des pieds au cou / prenons garde de ne pas perdre le reste pour qu'on puisse tenir en vie" LA LANGUE A ÉTÉ AVALÉE / avaler tu sais ce que ca veut dire avaler / [...].

Faty: avaler oui /

RPC : Avaler oui / ce qui veut dire qu'aujourd'hui la langue pulaar a été avalée / on a perdu beaucoup de choses dans cette langue / si tu constates aujourd'hui / de nos jours beaucoup d'expressions en pulaar sont assimilables au costume des Baay Faal $^{6}$ / c'est du njaxaas / donc le peu qu'il nous reste saisissons-le avant qu'il nous passe à travers les doigts / puisqu'elle a été avalée apprenons-la / en plus de nos jours le processus d'avalement des cultures s'annonce / des langues voisines des langues étrangères occidentales sont là / cela veut dire qu'on doit beaucoup faire pour notre culture / beaucoup faire aussi pour notre langue / dans la mesure où une ethnie ${ }^{7}$ c'est d'abord sa langue / si cette langue ne se parle plus cette ethnie meurt / » (Faty 2011: 334-335).

Cet extrait est tiré d'une conversation que j'ai eue avec un militant de la langue pulaar, par ailleurs, responsable des programmes culturels de la Radio communautaire de Matam (ibid. : «Ethnographie »). Dans cet extrait, on retrouve une approche biologisante de la langue avec la comparaison anthropomorphique que le militant fait de la langue pulaar : «/ notre langue a été avalée des pieds au cou /. » À travers cette phrase, se dévoile la métaphore de la langue-corps (Canut 2007a : 102), dotée de tous les organes (membres) à l'image du corps humain. Ce rapprochement entre la langue et le corps humain n'est pas gratuit dans ce type de discours, dont le fonctionnement repose largement sur la reprise d'autres théorisations culturalistes des langues, d'autant que leurs auteurs, généralement, sont intellectuellement bien armés pour aborder toutes les questions relatives à la défense de leur langue. L'interlocuteur — un intellectuel informé de l'actualité nationale et internationale sur tout ce qui a trait à la défense des minorités en est même venu à citer un rapport de l'Unesco prédisant la «mort» de

5. Le responsable des programmes culturels de la Radio communautaire de Matam.

6. Une branche de la confrérique mouride, (les Baay Faal) dont les disciples, pour faire œuvre d'ascétisme, se font confectionner des boubous à partir de morceaux de tissus qu'ils récupèrent un peu partout ou de chutes chez le tailleur. Ce qui donne à ce costume une forme de patchwork, de vêtement arlequin, qu'on appelle en wolof njaxaas. Le njaxaas est un mot wolof qui vient du verbe «jaxase», « mélanger».

7. Le mot leñol peut signifier ethnie, race, mais ici il peut aussi signifier communauté organisée de personnes ayant les mêmes intérêts. 
certaines langues, dans le but de légitimer sa vision apocalyptique des rapports entre les langues:

«Si tu regardes bien l'enquête qu'avait menée l'UNICEF euh non l'UNESCO / d'ici cinquante ans beaucoup de langues disparaîtront on ne les parlerait plus/ elles seront avalées par d'autres langues /» (Faty 2011: 334).

La langue, à partir de là, acquiert les mêmes propriétés et avantages (droits) que le corps auquel elle est assimilée. C'est ainsi qu'on tombe dans le fantasme de la pureté de la langue qui serait susceptible d'être souillée, salie au contact d'autres langues (maladies) venues de partout: «/ des langues voisines des langues étrangères occidentales, etc., sont là /. » On retrouve ici une représentation médicale des phénomènes langagiers qui consiste à traiter la question de la compréhension des phénomènes de contacts de langues à partir du paradigme du mélange. Le mélange devient nocif pour la langue puisqu'on suppose qu'il l'altère. Dans l'extrait cidessus, le militant pulaarophone essaie de rendre compte de ce phénomène qu'il croit être « un risque d'abâtardissement» de la langue pulaar à travers les emprunts que cette langue fait aux autres. Les contacts entre langues font l'effet d'une malédiction :

«[...] on a perdu beaucoup de choses dans cette langue [le pulaar] / si tu constates aujourd'hui / de nos jours beaucoup d'expressions en pulaar sont assimilables au costume des Baay Faal / c'est du njaxaas /.»

Le mot njaxaas utilisé ici montre tout le mépris que ce militant éprouve à l'égard du mélange. Si le vêtement njaxaas symbolise pour la communauté Baay $\mathrm{Faal}^{8}$ un choix de vie ascétique, il n'en demeure pas moins qu'il est souvent associé à la précarité, à la pauvreté dans l'imaginaire populaire. Au Sénégal, la métaphore de l'habillement est souvent utilisée pour parler des langues. Il existe un adage wolof qui dit:

« Kuy woddo lamiñ bu noppe rafle.

Si la langue est notre vêtement, l'on se dénude dès lors qu'on se tait » (dès lors qu'on ne la pratique pas).

C'est certainement cette peur d'être nu qui anime ce militant dans sa volonté de défendre sa langue. Il s'insurge contre l'hétérogénéité pourtant constitutive de la langue (Canut 2007a), en réactivant le fantasme de son altération et de sa dégénérescence par les autres langues. Le militant laisse planer la nostalgie d'une langue pulaar originelle et pure.

Quels sont les intérêts d'un tel positionnement? Dans ce processus d'essentialisation de la langue et de la culture haalpulaar, se profile un enjeu identitaire consistant à nommer et à définir son «autre » dans le but de

8. Voir la note 6 , page 71. 
créer une entité politique, communautaire et identitaire, dont la langue reste le marqueur principal.

« Donc le peu qui nous reste d'elle prenons-en soin avant qu'il nous échappe / vue qu'elle est avalée prenons-en soin / c'est le temps des cultures avalées / en plus les langues qui nous entourent celles qui traversent l'océan et bien d'autres sont à l'affût / cela veut donc dire que nous devons nous mobiliser pour notre culture / nous mobiliser aussi pour notre langue / parce que un peuple existe par sa langue / si cette langue n'est plus parlée ce peuple meurt / (Faty 2011:334).

On voit bien ici l'évolution du discours de ce militant qui devient de plus en plus marqué par un passage à l'action pour la défense de la pureté de la langue pulaar. En tant que promoteur de la langue pulaar, de par son statut de responsable de radio communautaire, le militant veille au maintien d'un pouvoir qui lui est essentiel : celui dont il tire la considération des populations, des autorités politiques locales et même nationales qui n'hésitent pas à passer par lui pour solliciter les suffrages. Souvenons-nous que Tidiane Anne (journaliste à la Radio télévision sénégalaise [RTS]), l'une des figures les plus charismatiques du mouvement de défense de la langue pulaar au Sénégal, a trouvé la mort par accident lors d'une tournée électorale que son candidat (Abdoulaye Wade) menait dans le Fouta. Lors de cette tournée électorale, il a été sollicité par son candidat pour l'aider à s'adresser aux populations du Fouta auprès de qui il jouissait d'une très grande popularité. Le discours homogénéisant et l'appel constant du militant à la mobilisation pour sa culture et sa langue sont souvent liés à une volonté de devenir un intermédiaire entre les instances de pouvoir et les populations locales.

\section{Quand la poésie sert à dire «non»}

La langue pulaar, à travers la littérature, devient une arme politique et culturelle pour faire passer non seulement des messages mais aussi pour construire un certain nombre de mythes (mythes attachés à la puissance d'antan du Fouta). En effet, la codification des langues nationales en général et du pulaar en particulier a permis aux militants de raconter des mythes historiques et contemporains, si l'on considère les mythes comme des actes de langage par lesquels les sujets interviennent dans l'histoire, autrement dit «[des] outil[s] d'expression politique» (Bensa 2006 : 131). Le mythe est « un langage de signes par lequel les hommes expriment leurs droits et leurs statuts » (ibid. : 129). Et les poèmes, dans la mesure où ils participent à la revendication d'un certain nombre de droits, figurent comme autant de pensées en acte qui ont pris « une coloration mythique» (Derive 2002 : 172).

Le poème, qui va être soumis à l'étude, a été diffusé à la fin d'une émission culturelle portant sur une affaire qui avait défrayé la chronique en mai 2009 à Matam. Quelques semaines auparavant, un père de famille venait d'être incarcéré pour avoir accepté de faire exciser sa fille. D'après 
les informations diffusées sur les radios, l'affaire avait été portée en justice par les associations de défense des droits de l'Homme comme la Raddho 9 . La rumeur évoquait quant à elle un « coup monté » par TOSTAN (une ONG qui lutte contre l'illettrisme et l'analphabétisation pour sensibiliser les populations rurales, surtout les femmes), avec le concours de l'État sénégalais, dans le but de «saper » le patrimoine culturel du Fouta. L'ONG TOSTAN, dans la mesure où elle s'investit beaucoup dans l'enseignement et l'apprentissage du pulaar en engageant des enseignants dans des formations pédagogiques, avait bénéficié jusqu'alors d'une sympathie énorme auprès des populations du Fouta. Or, ironie du sort, elle était accusée de vouloir participer à l' " anéantissement de cette culture ». C'est dans ce contexte particulier que la radio communautaire de Matam avait passé ce poème engagé à la fin d'une émission portant sur cette affaire.

\section{«Poème 1: Fuuta saa innama}

Fouta à chaque fois qu'on parle de toi

YiiYam lalla e mbeerdam

Mon sang m'irradie le cœur

Miijooji muusdi kodomi

Je deviens mélancolique

Dooyli jam ngeggami

Point de sommeil (je ne dors plus)

Aduna sooftami

Le monde s'affadit à mes yeux

Naamri mbelndi mettita

Point d'appétit

Haaju bona

Je ne sais point quoi faire

Mi haamto mi sab6ito

Et je suis pris de remords

Mi miijo haanki

Je pense au passé

Haanki momi tawaka

Ce passé que je n'ai point vécu

Haanki mo tindinami haande

Ce passé dont le compte m'est rendu qu'aujourd'hui

Sangay sangajii

Ce passé glorieux

Nde kolli mbelii mbebaani

Du temps où la musique était belle

Jammaji diNre

Où il y avait encore des veillées

Boombi e saggatabe

Où se mêlaient jeunes filles et éphèbes

Lewlewe demminare

Sous les pleines lunes des nuits d'été

Fuuta

Fouta

9. Rencontre africaine pour la défense des droits de l'Homme. 
Gila naange ma muti

Depuis que ton soleil s'est couché

Leewru ma roonki ummade

Impossible pour toi de voir poindre ta lune

Samba ari ngendiyen

Des étrangers surgis de nulle part

Ngari curri fiyaka moggi

Ont débarqué

Gannde ma njedda mbirni

C'est ainsi que tes savoirs sont occultés

Adaji puuyna cuudi

Tes coutumes ridiculisées et cachées

Ngaalu ma wujja

Ta richesse spoliée

Needagaal ma tama

Ton Etre amoindri »

(Faty 2011 : 351).

L'analyse stylistique, historique et interprétative de ce poème, ouvrira sur une étude des déictiques personnels (je et tu) en insistant sur leur caractère performatif, c'est-à-dire l'orientation du contenu vers les désirs ou vœux du poète (Gelongal $\mathrm{Ba}$ ) dont le seul objectif est d'intervenir sur le cours de l'histoire afin de rétablir un équilibre (Deprez 2002 : 45).

Ce poème met en évidence diverses composantes pertinentes pour les propos que je voudrais développer ici. D'abord, tout au long de ces vers, la question du fantasme de l'adhésion du sujet à un seul et unique territoire est posée. Ensuite, ce qui y est mis aussi en évidence, c'est l'existence d'une altération d'un «passé glorieux » du Fouta, en un présent maussade : c'est ce qui plonge l'auteur, dans le désarroi. Derrière le déictique personnel je de l'auteur se cachent ses autres semblables, qu'il doit essayer de «toucher », dans le but de leur faire prendre conscience de la "gravité » de la situation. L'auteur joue donc sur la dimension empathique du langage, ce qui donne à ce poème une visée propagandiste en réaction à un « malaise », un « déséquilibre » (Deprez 2002), à une « atteinte à l'intégrité » culturelle, politique, et même économique du Fouta. Le déictique personnel je, par ailleurs sujet de l'énonciation, renferme une portée indexicale et performative dans le sens où il essaie d'agir et de faire agir, d'intervenir sur les comportements des autres dont la somme constituerait une seule et même entité. On retrouve ici le fantasme de l'unité territoriale et du « recueillement dans le un» (Canut 2007a: 16).

La présence du déictique $t u$ dans ce poème est quant à elle, un moyen pour l'auteur de s'adresser à la fois aux lecteurs/auditeurs et au Fouta, à travers le procédé de la personnification. Le territoire ainsi que les auditeurs sont ainsi constamment apostrophés tout le long de ce poème au point de former une même unité. En effet, en s'adressant aux lecteurs, l'auteur utilise une figure de style appelée « optation » qui, selon P. Fontanier (1977 : 438), « est l'expression d'un désir ardent d'obtenir pour soi ou pour d'autres 
quelque chose à quoi l'on attache, au moins pour le moment, un prix et une grande importance». L'auteur, voulant «engager» les habitants du Fouta dans son combat pour la défense de la culture haalpulaar, les interpelle, les apostrophe (à l'aide de la deuxième personne du singulier) en faisant l'étalage des dommages causés par «les envahisseurs »: «[...] tes savoirs sont occultés, tes coutumes ridiculisées, etc. » Mais les frontières de l'objet désiré semblent floues dans la mesure où le déictique $t u$ confond le territoire et les habitants. Si l'optation avait pour rôle de dévoiler un vœu, la personnification du territoire (Fouta) a, quant à elle, lieu dans ce poème, par le processus de métonymisation, et ce, dans le but de réduire ou d'assimiler l'espace aux occupants.

Le discours véhiculé par ce poème veut sensibiliser les Haalpulaar car, comme le poète, beaucoup de militants de la cause pulaar déplorent la « léthargie » dans laquelle la population haalpulaar est plongée, refusant par exemple d'apprendre le pulaar, ou de participer au financement de certains projets. Si certains militants le suggèrent à travers l'art comme ici la poésie, d'autres, comme ce militant enseignant de pulaar, se désolent ouvertement de l'inaction de leur communauté :

« Nous autres nous avons qu'un seul problème / nous avons des savants nous avons aussi des personnes riches / mais ils n'ont pas voulu y mettre leur argent / leur savoir-faire pour que la langue puisse se développer / (Faty 2011 : 340-341).

L'auteur du poème, pour sortir de l'état de désolation que lui inspire la situation actuelle du Fouta, procède à une célébration, non sans nostalgie d'ailleurs, du passé de son « pays » comme si ce dernier devait y trouver son salut. Il ne se contente que de métaphores astrales pour décrire la déchéance du Fouta.

\author{
«Fuuta \\ Fouta \\ Gila naange ma mutii \\ Depuis que ton soleil s'est couché \\ Leewru ma roonki ummade \\ Impossible pour toi de voir poindre ta lune.»
}

La figure du responsable est omniprésente dans ce genre de production discursive, mais là aussi, impossible d'obtenir davantage d'informations sur l'identité du responsable qu'on se contente seulement de nommer par des syntagmes aussi vagues que «étrangers» ou «ennemis ». Un flou entoure cette figure de l'altérité contagieuse ou destructrice au point de faire divaguer l'imagination du poète. En suivant la conception foucaldienne du langage, il apparaîtra que l'essentiel ici, n'est pas de connaître le sens que manifeste ou cache ce poème (discours), mais l'objet même de cet événement, c'est-à-dire selon Foucault (1971: 12) «ce pour quoi, ce par quoi on lutte ». Quelles que soient les raisons d'un tel déclin, nous constatons 
dans ce poème la présence d'une posture victimaire de l'auteur, qui cherche à rassembler, à fédérer autour de lui afin de créer une communauté de victimes susceptibles de revendiquer un certain nombre de «droits » comme par exemple celui à la différence, celui de protéger et de sauvegarder son terroir, sa langue et sa culture, etc.

L'étude de ce poème confirme donc la thèse de la chercheure Mélanie Bourlet (2007) qui, dans un de ces travaux, a affirmé que la poésie pulaar a une double fonction: didactique et contestataire. La spécialiste de la poésie en langue pulaar reconnaît également le caractère éminemment politique des poèmes qui trouvent leur ancrage dans un contexte politique bien particulier.

«La poésie pulaar est avant tout une poésie engagée, dont l'émergence et le contenu sont liés au contexte politique des années 70 et 80 dans ces deux pays [le Sénégal et la Mauritanie]»(Bourlet $2007: 38$ ).

\section{La question du pouvoir au centre des revendications}

«Allah jarama a jarama / rendo ngo fof weltanimama / njokke goolle no haanirtani / Allah wallu en / haa leñol ngol LAAMO e LAAMU mo hay goto heptata.

Je te remercie beaucoup / toute la communauté (les auditeurs) est contente / je vous souhaite de redoubler d'efforts et de courage / Dieu fasse que / pour la communauté le POUVOIR soit de notre côté / un POUvoIR que l'on ne saurait ravir/» (Faty 2011: 348).

Après avoir apporté une contribution à un thème choisi lors d'une émission culturelle radiophonique, un auditeur a formulé sans ambages ses vœux (ci-dessus), prononçant enfin le mot «pouvoir» dans son discours, contrairement à beaucoup de militants qui n'utilisent jamais de manière explicite ce mot, alors même que l'on peut conjecturer sans se tromper que tout tourne autour de lui.

La mise en scène de l'identité haalpulaar, à travers la défense de son patrimoine culturel, linguistique et territoriale, s'inscrit, comme la plupart des mouvements identitaires, dans une logique de pouvoir. La question politique apparaît comme un élément central pour comprendre la finalité de ces mouvements. En effet, dans les discours recueillis sur le terrain, j'ai souvent constaté qu'il est davantage question de stratégies mises en place afin d'améliorer les conditions de vie de toute une population rurale (celle du Fouta) qui se sent abandonnée par les pouvoirs publics. Il s'agit de défendre « une dignité » supposée perdue en magnifiant, par exemple, l'histoire du peuple du Fouta qui, face à l'évolution politique, économique et sociale, a perdu sa position d'antan. Ou alors de se faire le porte-parole de tout un peuple en faisant remonter les revendications portant sur des questions aussi vitales que la politique des terres, la pauvreté, la désertification, etc., ce qui est une manière de ne pas se faire oublier par le «pouvoir central ». 
On assiste à une instrumentalisation politique des questions linguistiques à l'œuvre dans les discours des militants, comme par exemple celui du chargé des programmes de la Radio communautaire de Matam que je soumettrai à l'analyse. En effet, quand je lui ai posé la question de savoir qui était Tidiane Anne (un défenseur de la langue et de la culture pulaar, un ngendiyanke, comme on l'appellerait dans le milieu, par ailleurs journaliste culturel), et le rôle qu'il jouait au sein de la «communauté » haalpulaar, il n'a pas tardé à m'énumérer ses bienfaits à l'égard des populations du Fouta, des bienfaits qui se résument à des actions politiques ciblées et concrètes :

«Parmi ces projets il y avait l'instauration d'une Radio Fulfulde / afin que tous les Fulbe puissent se retrouver / il y avait aussi des projets de développement / il s'était battu pour tout ce qui est de l'"après-barrage" et aussi pour le "domaine national" / et son idée consistait à dire pourquoi on ne commence pas par appliquer le principe du domaine national à Dakar plutôt qu'ici ${ }^{10} /$ tout ceci c'est comme si on n'avait personne qui serait susceptible de le relever et de l'achever / c'est comme s'il n'y avait pas de relais/» (Faty $2011: 331$ ).

Afin de comprendre cet extrait où l'on voit apparaître des questions purement politiques, un point historique et contextuel s'impose pour faciliter la compréhension de ce discours, ce qui permettra de mettre au jour l'imbrication des intérêts discursifs en fonction des données politiques, économiques et sociales que présente le contexte. Je vais donc entreprendre une « démarche explicative » pour essayer de comprendre les tenants et les aboutissants des énoncés qui composent les propos de ce militant. Selon Ducrot et al. (1980 : 22), «attribuer un sens à un énoncé, [...] c'est chercher pourquoi l'énoncé a été produit».

Il convient tout d'abord de rappeler que dans les années 1970, à la suite d'une explosion démographique et d'une sécheresse, deux barrages ont été construits le long du fleuve Sénégal pour faire face au déséquilibre économique, écologique et social survenu dans la moyenne vallée du fleuve (le Fouta). Le premier est situé à l'embouchure du fleuve Sénégal - c'est un barrage anti-sel (barrage Diama) —, et le second à $1500 \mathrm{~km}$ en amont (le barrage Manantali). Le terme d' «après-barrage» est un mot créé par les hommes politiques d'alors, pour prédire des lendemains meilleurs des habitants de cette zone qui pourront bénéficier des retombées économiques de la mise en valeur du fleuve. Le mot «après-barrage» était donc chargé d'optimisme pour tout habitant de cette zone. On a toujours entendu dire que l'«après-barrage » (entendons après la mise en place des barrages), la vallée du fleuve serait le «grenier du Sénégal». Mais cette époque de prospérité a été repoussée aux calendes grecques : l' «après-barrage » tient

10. Entendons dans le Fouta, ce qui sous-tend qu'on est non seulement dans la défense d'une langue-identité mais aussi d'un territoire-terroir. 
toujours lieu de promesse. Pour la plupart des habitants de cette zone, cette inefficacité à faire fructifier ce projet économique naît d'une asthénie gouvernementale et d'un manque d'engagement pour cette zone non wolofone. Mamadou Diouf (2001 : 84), reconnaît même que « les perspectives de l'après-barrage et l'accès à des terres mises en valeur grâce à l'endettement de l'État, qui veut rentabiliser son investissement», sont parmi les «événements importants [qui] devront peser dans l'évolution des Puular/ Haalpulaar».

Si l'auteur fait ensuite référence au «domaine national », c'est qu'il y a eu en 1964 une loi au Sénégal, portant sur le domaine national, prévoyant une privatisation des terres : la loi 64-46 du 17 juin 1964. C'est une loi qui s'est également inspirée du socialisme africain prôné par l'ancien président, Senghor, lequel affirmait, à propos de cette loi, qu' « il s'agit de revenir du droit romain au droit négro-africain, de la conception bourgeoise de la propriété foncière à la conception socialiste qui est celle de l'Afrique noire traditionnelle » (cité dans Sow 1997 : 55). Il s'est agi de soustraire la terre à une propriété coutumière et de la socialiser, ce qui ne manquait pas de créer un malentendu et de susciter de vives oppositions chez les paysans du Fouta, en raison du lien sacré entre la terre et ces derniers. D'autant plus que dans cette zone du Sénégal s'est créée, au fil des ans, une aristocratie foncière qui a développé un mythe reposant sur un branchement entre l'identité haalpulaar et les terres irriguées par le fleuve. Ainsi, tout Haalpulaar est-il fier des terres du waalo (terres fertiles irriguées par les eaux du Sénégal), qu'il considère comme partie intégrante de son identité et est prêt à s'engager pour les défendre. La défense des terres du waalo s'expliquerait par une volonté politique de conserver un des «trois systèmes de pouvoir » (Schmitz 1994 : 419), qui prévaut dans le Fouta. Comme tout système de pouvoir, l'organisation sociale et politique de la société (en l'occurrence la société haalpulaar) «se projette sur le dispositif » (ibid: 421). En effet, selon Schmitz (ibid.), la répartition et le contrôle des terres du waalo, sont fortement liés à la hiérarchisation sociale de la société haalpulaar, notamment du rang social occupé. Remettre en question ce système reviendrait de ce fait, aux yeux des militants, à saper l'équilibre et les traditions de la cité haalpulaar, partant de son identité. D'où la présence de cette question à côté des revendications linguistiques.

La référence faite par l'auteur à ces deux faits témoigne donc de l'orientation sociopolitique des discours sur l'identité haalpulaar. Dans ces deux exemples, l'imbrication discursive entre les questions politiques, économiques et identitaires est directe et explicite. La complexité formelle de ces discours identitaires n'est pas aussi évidente à détecter chez les militants qui, la plupart du temps «avancent masqués ».

Comme on l'a vu, la question du pouvoir est donc au centre des revendications identitaires des Haalpulaar dans lesquelles des questions de partage des richesses, de développement, de droits reviennent tel un leitmotiv. 
«Les gens doivent s'unir / ils doivent aussi s'entraider / s'aimer les plus riches doivent aider les plus démunis / ils doivent aussi comprendre que les droits ça s'arrache / si t'es dans un pays attendant qu'on te donne tes droits tu auras rien $\mathrm{du}$ tout / tout ce que nous devons avoir comme droit nous devons l'arracher / arracher ne va pas dire non plus une guerre / mauvaise idée / (Faty 2011 : 344).

Ces propos sont extraits d'une discussion de Tidiane Anne (journaliste et militant charismatique du mouvement pulaar du Fouta, mort en 2001), disponible en cassette audio. Il fut l'un des plus grands théoriciens et porte-parole de ce mouvement ${ }^{11}$. Il avait le «droit privilégié et exclusif» (Foucault 1971 : 11) de parler pour cette «communauté » au point de devenir un véritable promoteur de la langue et de la culture pulaar.

À travers cet article, nous avons essayé de passer en revue les différentes formes d'homogénéisation dont font l'objet la langue et la culture haalpulaar au Sénégal. En interrogeant les discours des militants, nous avons rendu compte du poids des enjeux politiques dans le combat pour la défense de la culture et de la langue pulaar.

Dans un des articles de l'ouvrage ${ }^{12}$ consacré aux différentes facettes de politique d'émancipation linguistique, publié dans la revue International Journal of the Sociology of Language, L. Huus et A. R. Lindgren (2011), s'interrogeaient d'emblée sur la connexion entre langue et émancipation. Selon eux, ce sont les structures et les hiérarchies de pouvoir qui définissent les relations entre les langues dans une communauté donnée. Ce qui veut dire que certaines langues jouissent de statuts plus favorables que d'autres. Par conséquent, la langue, comme l'ont affirmé Huus et Lindgren (ibid.), devient dès lors un moyen pour renverser ces hiérarchies de pouvoir, notamment dans les sociétés multilingues. C'est ainsi qu'ils définissent l'émancipation linguistique comme une amélioration de la position de la langue minorée, dominée ou «mal classée ». Et ce, à travers un certain nombre d'actions et de discours dans le but de briser les structures d'inégalités.

Dans le cas de cet article, l'analyse des discours ainsi que des productions artistiques (poèmes) des militants, rend compte à quel point les questions de pouvoir sont donc au centre des actions et des discours des militants pulaarophones. La langue pulaar n'est de ce fait qu'un instrument, un prétexte, un faire-valoir pour tenter de renverser des hiérarchies de pouvoir (politique et économique) dans un pays où la langue wolof est vue de plus en plus comme une langue hégémonique.

11. Les discussions de ce dernier sur la langue pulaar ou sur les Haalpulaar sont souvent compilées dans des supports médiatiques et vendues dans des boutiques spécialisées.

12. Cet ouvrage a été coordonné par L. HuUs et J. Shaun-Nolan (2011). 
Cependant, il n'existe pas que des enjeux politiques et économiques dans le processus de mise en discours du Fouta, de ses habitants et de sa langue. En effet, au-delà de l'aspect politique, on retrouve également un certain nombre d'enjeux symboliques qui pourraient faire l'objet d'une étude particulière.

Centre de recherche sur les liens sociaux (CERLIS), Université Paris Descartes, Paris.

\section{BIBLIOGRAPHIE}

Augé, M.

2009 Pour une anthropologie de la mobilité, Éditions Payot \& Rivages.

BENSA, A.

2006 La fin de l'exotisme: essai d'anthropologie critique, Toulouse, Anacharsis Éditions.

BOURLET, M.

2007 «Poésie pulaar: politique dans les années 70 et 80 au Sénégal et en Mauritanie », in Littérature savoirs et enseignement, Centre d'études linguistiques et littéraires francophones et africaines, Bordeaux, Presses de l'Université de Bordeaux : 37-46.

Boutet, J.

2008 La vie verbale au travail, des manufactures aux centres d'appels, Toulouse, Octares.

CANUT, C.

2007a Une langue sans qualité, Limoges, Éditions Lambert-Lucas.

2007b «L'épilinguistique en question», Sciences pour la communication, 81 : 49-72.

2008a Le spectre identitaire : entre langue et pouvoir au Mali, Limoges, Éditions Lambert-Lucas.

2008 b « Des grains dansant dans la poussière du visible. Imaginaire linguistique et sujet parlant», Sêméion, 7, «Imaginaire linguistique et imaginaire culturel $\gg: 75-86$.

DAFF, M.

1996 «Appropriation du français, particularités lexicales et indices de territorialité d'un texte littéraire », Sciences \& Techniques du Langage, 2 (juin) CLAD, Dakar : 29-47. 
DEPREZ, C.

2002 «La langue comme "épreuve" dans les récits de migration », Bulletin suisse de linguistique appliquée, 76 : 39-52.

Derive, J.

2002 L'épopée et la diversité d'un genre, Paris, Karthala.

Diouf, M.

1994 Sénégal : les ethnies et la Nation, Paris, L'Harmattan.

2001 L’histoire du Sénégal: le modèle islamo-wolof et ses périphéries, Paris, Maisonneuve \& Larose.

Dreyfus, M. \& Julliard, C.

2001 «Le jeu de l'alternance dans la vie quotidienne des jeunes scolarisés à Dakar et à Ziguinchor : variation dans l'usage du français et du wolof », Cahiers d'Études africaines, XLI (3-4), 163-164 : 667-696.

DUCROT, O. ET AL.

1980 Les mots du discours, Paris, Les Éditions de Minuit.

Ducrot, O. \& SchaEFFER, J.-M.

1995 [1972] Nouveau dictionnaire encyclopédique des sciences du langage, Paris, Éditions du Seuil.

FAty, E. A. A.

2011 Processus d'homogénéisation linguistique et instrumentalisations discursives au Sénégal: le cas des Haalpulaar, Thèse de doctorat, Paris, Université Paris Descartes.

FONTANIER, P.

1977 Les figures du discours, Paris, Flammarion.

Foucault, M.

1971 L'ordre du discours, Paris, Gallimard.

Huss, L. \& Lindgren, A. R.

2011 «Introduction : Defining Language Emancipation», in L. Huss \& J. SHAunNolan (eds.), The Many Face of Language Emancipation, International Journal of the Sociology of Language, 209: 1-15.

Mc Laughlin, F.

1995 «Haalpulaar Identity as a Response to Wolofization», African Language and Culture, 8 (2) : 153-168.

2001 «Dakar Wolof and the Configuration of Urban Identity », Journal of African Cultural Studies, 14 (2) : 153-172.

2009 «Wolof as an Urban Vernacular in Senegal», in C. B. Vigouroux \& S. S. Mufwene (eds.), Globalization and Language Vitality. Perspectives from Africa, London, A\&C Black: 142-170. 
NDAO, P. A.

1996 Contacts de langues au Sénégal, étude du code-switching wolof/français en milieu urbain: approches linguistique, sociolinguistique et pragmatique, Thèse de doctorat, Dakar, Université Cheikh Anta Diop.

2009 «Toute langue a une durée de vie », Entretien, Le Quotidien, 8 juillet.

NDIAYE, M.

1996 L'éthique ceddo et la société d'accaparement ou les conduites culturelles des Sénégalais d'aujourd'hui, t. 1, Le Goorgi, t. 2, Les Moodu Moodu, Dakar, Presses universitaires de Dakar.

O'Brein, D. C.

1998 «The Shadow-Politics of Wolofisation», The Journal of Modern African Studies, 36 (1) : 25-46.

O’Brein, D. C., Diop, M. C. \& Diouf, M.

2002 La construction de l'État du Sénégal, Paris, Khartala.

Schmitz, J.

1994 «Cités noires : les républiques villageoises du Fuuta Tooro (Vallée du fleuve Sénégal) », Cahiers d'Études africaines, XXXIV (1-2-3), 133-135 : 419-460.

SMITH, É.

2009 «Un modèle républicain pluraliste ? Les trajectoires ambiguës de l'idéologie républicaine au Sénégal », Congrès AFPS 2009, Section thématique 9, Républiques, trajectoires historicités et voyage d'un concept (texte provisoire), <http://www.congresafsp2009.fr/sectionsthematiques/st9/st9smith.pdf> (consulté le 6 décembre 2014).

2010 «La nationalisation par le bas : un nationalisme banal? Le cas de la wolofisation au Sénégal », Raisons politiques, 37:65-77.

Sow, A. S.

1997 «Domaine national, la Loi et le Projet de Réforme», La Revue du Conseil économique et social, 2, février-avril : 55-65.

Tine, A.

1985 « Sembène et son rapport à la langue de communication littéraire. La prééminence objective du français et la prééminence subjective du wolof », Annales de la faculté des Lettres et Sciences humaines de Dakar, 15 : 207-220.

Vigouroux, C.

2003 Réflexion méthodologique autour de la construction d'un objet de recherche: la dynamique identitaire chez les migrants africains francophones au Cap (Afrique du Sud), t. 1, Thèse de doctorat, Nanterre, Université de Paris XNanterre.

2009 «From Africa to Africa : Globalization, Migration and Language Vitality», in C. B. Vigouroux \& S. S. Mufwene (eds.), op. cit. : 229-254. 


\section{RÉSUMÉ}

Cet article propose d'étudier et d'analyser les discours épilinguistiques des militants pulaarophones du Sénégal. Il s'agit plus précisément de rendre compte de l'instrumentalisation discursive dont la langue pulaar fait l'objet, à travers divers processus d'homogénéisation, à des fins politiques, économiques et sociales. D'un point de vue méthodologique, cette étude s'inscrit dans le cadre de l'anthropologie des pratiques langagières, et adopte donc une approche pluridisciplinaire.

\section{ABSTRACT}

The "Haalpulaarization" or the "Enwordment" of Pulaar Language and Culture in Senegal. Processes and Stakes. - This article proposes to explore and analyze the epilinguistic discourses of pulaarophone Senegalese activists. More specifically, it involves clarifying the discursive intrumentalization that Pulaar language provides through diverse homogeneization processes, for social, economic and political purposes. From a methodological point of view, this study fits within the framework of the anthropology of language practices, thus it adopts a multidiciplinary approach.

Mots-clés/Keywords : Sénégal, haalpularisation, homogénéisation linguistique, instrumentalisation discursive, langues et identités, wolofisation/Senegal, haalpularization, linguistic homogeneization, discursive instrumentalization, languages and identities, wolofization. 